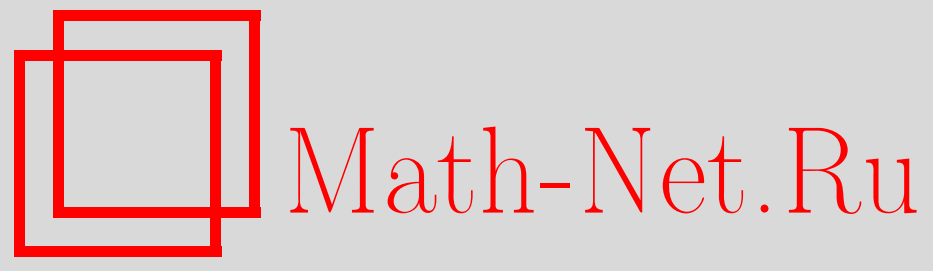

К. Б. Сабитов, Асимптотические оценки разностей произведений функций Бесселя на интеграл от этих функций, Вестн. Сам. гос. техн. ун-та. Сер. Физ.-мат. науки, 2020, номер 1, 41-55

DOI: https://doi.org/10.14498/vsgtu1685

Использование Общероссийского математического портала MathNet.Ru подразумевает, что вы прочитали и согласны с пользовательским соглашением http://www . mathnet.ru/rus/agreement

Параметры загрузки:

IP: 52.205 .19 .152

26 апреля 2023 г., 15:48:13

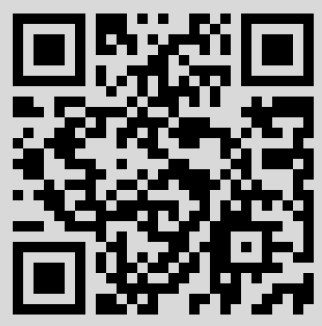


Вестн. Сам. гос. техн. Ун-та. Сер. Физ.-мат. науки. 2020. Т. 24, № 1. С. 41-55 ISSN: 2310-7081 (online), 1991-8615 (print)

УДК $517.95+517.584$

\title{
Асимптотические оценки разностей произведений функций Бесселя на интеграл от этих функций
}

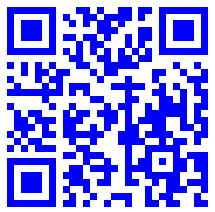

\author{
К. Б. Сабитов \\ 1 Стерлитамакский филиал Башкирского государственного университета, \\ факультет математики и информационных технологий, \\ Россия, 453103, Стерлитамак, проспект Ленина, 37. \\ 2 Институт стратегических исследований Республики Башкортостан, \\ Стерлитамакский филиал, \\ Россия, 453103, ул. Одесская, 68.
}

\begin{abstract}
Аннотация
При исследовании прямых и обратных задач по отысканию правой части вырождающихся уравнений смешанного типа с различными граничными условиями возникает задача об установлении асимптотических оценок для разностей произведений цилиндрических функций на интеграл от этих функций. Предварительно на основании установленной новой формулы нахождения конечной биномиальной суммы вычислены разности произведений цилиндрических функций на определенный интеграл от этих функций через обобщенную гипергеометрическую функцию. С использованием асимптотической формулы при больших значениях аргумента для обобщенной гипергеометрической функции установлены асимптотические оценки при больших значениях параметра для указанных разностей функций Бесселя первого и второго рода, а также для модифицированных функций Бесселя.
\end{abstract}

Ключевые слова: функции Бесселя, модифицированные функции Бесселя, интегралы от функций Бесселя, конечная биномиальная сумма, обобщенная гипергеометрическая функция, асимптотические оценки.

Получение: 3 апреля 2019 г. / Исправление: 16 августа 2019 г. Принятие: 16 сентября 2019 г. / Публикация онлайн: 21 ноября 2019 г.

\section{Научная статья}

(2) (7) Контент публикуется на условиях лицензии Creative Commons Attribution 4.0 International (https://creativecommons.org/licenses/by/4.0/deed.ru)

\section{Образец для цитирования}

Сабитов К. Б. Асимптотические оценки разностей произведений функций Бесселя на интеграл от этих функций // Вестн. Сам. гос. техн. ун-та. Сер. Физ.-мат. науки, 2020. T. 24, № 1. C. 41-55. doi: 10.14498/vsgtu1685.

\section{Сведения об авторе}

Камиль Басирович Сабитов (1) https://orcid.org/0000-0001-9516-2704

доктор физико-математических наук, профессор; заведующий кафедрой, каф. математического анализа ${ }^{1}$; зав. лабораторией, лаб. прикладной математики и информатики ${ }^{1}$;

e-mail: sabitov_fmf@mail.ru 
Введение. При исследовании обратных задач по отысканию правой части вырождающихся уравнений смешанного типа [1-10] возникает задача об установлении асимптотических оценок для следующих разностей:

$$
\begin{gathered}
N_{\nu}^{(1)}(y)=\sqrt{y} I_{\nu}\left(p y^{q}\right) \int_{0}^{y} \sqrt{s} I_{-\nu}\left(p s^{q}\right) d s-\sqrt{y} I_{-\nu}\left(p y^{q}\right) \int_{0}^{y} \sqrt{s} I_{\nu}\left(p s^{q}\right) d s \\
N_{\nu}^{(2)}(y)=\sqrt{y} I_{\nu}\left(p y^{q}\right) \int_{0}^{y} \sqrt{s} K_{\nu}\left(p s^{q}\right) d s-\sqrt{y} K_{\nu}\left(p y^{q}\right) \int_{0}^{y} \sqrt{s} I_{\nu}\left(p s^{q}\right) d s, \quad y>0 \\
S_{\nu}^{(1)}(y)=\sqrt{-y} J_{\nu}\left(p(-y)^{q}\right) \int_{0}^{y} \sqrt{-s} J_{-\nu}\left(p(-s)^{q}\right) d s- \\
-\sqrt{-y} J_{-\nu}\left(p(-y)^{q}\right) \int_{0}^{y} \sqrt{-s} J_{\nu}\left(p(-s)^{q}\right) d s \\
S_{\nu}^{(2)}(y)=\sqrt{-y} J_{\nu}\left(p(-y)^{q}\right) \int_{0}^{y} \sqrt{-s} Y_{\nu}\left(p(-s)^{q}\right) d s- \\
-\sqrt{-y} Y_{\nu}\left(p(-y)^{q}\right) \int_{0}^{y} \sqrt{-s} J_{\nu}\left(p(-s)^{q}\right) d s, \quad y<0
\end{gathered}
$$

при $p \rightarrow+\infty$, где $\nu=1 /(2 q)=1 /(m+2), m>0$ - показатель степени вырождения уравнения, $J_{ \pm \nu}(\cdot)$ и $Y_{\nu}(\cdot)$ - функции Бесселя соответственно первого и второго рода, $I_{ \pm \nu}(\cdot)$ и $K_{\nu}(\cdot)$ - модифицированные функции Бесселя, $p=$ const $>0$.

Предварительно заметим, что

$$
\begin{gathered}
\sqrt{y} I_{ \pm \nu}\left(p y^{q}\right)=p^{-1 /(2 q)}\left(p y^{q}\right)^{1 /(2 q)} I_{ \pm \nu}\left(p y^{q}\right)=p^{-\nu} x^{\nu} I_{ \pm \nu}(x) \\
\sqrt{s} I_{ \pm \nu}\left(p s^{q}\right)=p^{-\nu} t^{\nu} I_{ \pm \nu}(t)
\end{gathered}
$$

где $x=p|y|^{q}, t=p|s|^{q}$,

$$
\int_{0}^{y} \sqrt{s} I_{ \pm \nu}\left(p s^{q}\right) d s=\frac{1}{q p^{3 \nu}} \int_{0}^{x} t^{3 \nu-1} I_{ \pm \nu}(t) d s
$$

Тогда разность (1) принимает вид

$$
N_{\nu}^{(1)}(y)=\frac{x^{\nu}}{q p^{4 \nu}} \widetilde{N}_{\nu}^{(1)}(x)
$$

Здесь

$$
\tilde{N}_{\nu}^{(1)}(x)=I_{\nu}(x) \int_{0}^{x} t^{\mu} I_{-\nu}(t) d t-I_{-\nu}(x) \int_{0}^{x} t^{\mu} I_{\nu}(t) d t, \quad \mu=3 \nu-1 .
$$

Аналогично (5) разности (2)-(4) представим в следующем виде:

$$
\begin{gathered}
N_{\nu}^{(2)}(y)=\frac{x^{\nu}}{q p^{4 \nu}} \widetilde{N}_{\nu}^{(2)}(x) \\
\tilde{N}_{\nu}^{(2)}(x)=I_{\nu}(x) \int_{0}^{x} t^{\mu} K_{\nu}(t) d t-K_{\nu}(x) \int_{0}^{x} t^{\mu} I_{\nu}(t) d t
\end{gathered}
$$




$$
\begin{gathered}
S_{\nu}^{(1)}(y)=-\frac{x^{\nu}}{q p^{4 \nu}} \widetilde{S}_{\nu}^{(1)}(x), \\
\widetilde{S}_{\nu}^{(1)}(x)=J_{\nu}(x) \int_{0}^{x} t^{\mu} J_{-\nu}(t) d t-J_{-\nu}(x) \int_{0}^{x} t^{\mu} J_{\nu}(t) d t \\
S_{\nu}^{(2)}(y)=-\frac{x^{\nu}}{q p^{4 \nu}} \widetilde{S}_{\nu}^{(2)}(x), \\
\widetilde{S}_{\nu}^{(2)}(x)=J_{\nu}(x) \int_{0}^{x} t^{\mu} Y_{\nu}(t) d t-J_{\nu}(x) \int_{0}^{x} t^{\mu} Y_{\nu}(t) d t, \quad x \geqslant 0 .
\end{gathered}
$$

Отметим, что разность (10) представляет собой функцию Ломмеля [11], $[12, \S 10.7],[13, \S 7.5 .5]$.

В данной работе, с использованием разложения функций $I_{ \pm \nu}(x)$ и $J_{ \pm \nu}(x)$ в степенной ряд, аналогично работам [14, 15], найдены разности (6) и (10) на основании установленной нами новой формулы для вычисления конечной суммы (см. лемму ниже), затем на их основе найдены (8) и (12). Отсюда в силу представлений $(5),(7),(9)$ и (11) получены окончательные результаты по разностям (1)-(4) и установлены асимптотические оценки при $p \rightarrow+\infty$.

1. Вычисление разностей (1)-(4). Используя разложения функций $I_{ \pm \nu}(t)$ в степенной ряд, предварительно найдем

$$
\begin{gathered}
\int_{0}^{x} t^{\mu} I_{\mp \nu}(t) d t=\int_{0}^{x} t^{\mu} \sum_{k=0}^{\infty} \frac{(t / 2)^{2 k \mp \nu} d t}{k ! \Gamma(\mp \nu+k+1)}= \\
=\sum_{k=0}^{\infty} \frac{x^{2 k+\mu \mp \nu+1}}{2^{2 k \mp \nu} k ! \Gamma(\mp \nu+k+1)(2 k+\mu \mp \nu+1)}
\end{gathered}
$$

и вычислим произведение рядов

$$
\begin{aligned}
& I_{ \pm \nu}(x) \int_{0}^{x} t^{\mu} I_{\mp \nu}(t) d t= \\
& =\sum_{k=0}^{\infty} \frac{(x / 2)^{2 k \pm \nu}}{k ! \Gamma( \pm \nu+k+1)} \sum_{m=0}^{\infty} \frac{x^{\mu+1}(x / 2)^{2 m \mp \nu}}{m ! \Gamma(\mp \nu+k+1)(2 m+\mu \mp \nu+1)}= \\
& \quad=x^{\mu+1} \sum_{k=0}^{\infty}\left(\frac{x}{2}\right)^{2 k} \sum_{n=0}^{k} \frac{[2(k-n)+\mu+1 \mp \nu]^{-1}}{n !(k-n) ! \Gamma( \pm \nu+n+1) \Gamma(\mp \nu+k-n+1)},
\end{aligned}
$$

где $\Gamma(\cdot)$ - гамма-функция Эйлера. Теперь составим их разность:

$$
\tilde{N}_{\nu}^{(1)}(x)=x^{\mu+1} \sum_{k=0}^{\infty}\left(\frac{x}{2}\right)^{2 k}\left(N_{1 k}-N_{2 k}\right) .
$$

Здесь

$$
N_{1 k}=\sum_{n=0}^{k} \frac{1}{n !(k-n) ! \Gamma(\nu+n+1) \Gamma(-\nu+k-n+1)(2 k-2 n+\mu+1-\nu)},
$$




$$
N_{2 k}=\sum_{n=0}^{k} \frac{1}{n !(k-n) ! \Gamma(-\nu+n+1) \Gamma(\nu+k-n+1)(2 k-2 n+\mu+1+\nu)} .
$$

В силу формулы для биномиальных коэффициентов

$$
\frac{\Gamma(z+1)}{\Gamma(\omega+1) \Gamma(z-\omega+1)}=\left(\begin{array}{c}
z \\
\omega
\end{array}\right)
$$

конечные суммы (14) и (15) представим в виде

$$
\begin{aligned}
& N_{1 k}=\frac{1}{(k !)^{2}} \sum_{n=0}^{k} \frac{1}{2(k-n)+\mu+1-\nu}\left(\begin{array}{l}
k \\
n
\end{array}\right)\left(\begin{array}{c}
k \\
n+\nu
\end{array}\right), \\
& N_{1 k}=\frac{1}{(k !)^{2}} \sum_{n=0}^{k} \frac{1}{2(k-n)+\mu+1+\nu}\left(\begin{array}{l}
k \\
n
\end{array}\right)\left(\begin{array}{c}
k \\
n-\nu
\end{array}\right) .
\end{aligned}
$$

На основании равенства

$$
\left(\begin{array}{l}
a \\
b
\end{array}\right)=\left(\begin{array}{c}
a \\
a-b
\end{array}\right)
$$

преобразуем конечные суммы (16) и (17):

$$
\begin{gathered}
N_{1 k}=\frac{1}{(k !)^{2}} \sum_{n=0}^{k} \frac{1}{2(k-n)+\mu+1-\nu}\left(\begin{array}{c}
k \\
k-n
\end{array}\right)\left(\begin{array}{c}
k \\
k-n-\nu
\end{array}\right)= \\
=\frac{1}{(k !)^{2}} \sum_{l=0}^{k} \frac{1}{2 l+\mu+1-\nu}\left(\begin{array}{c}
k \\
l
\end{array}\right)\left(\begin{array}{c}
k \\
l-\nu
\end{array}\right), \\
N_{2 k}=\frac{1}{(k !)^{2}} \sum_{l=0}^{k} \frac{1}{2 l+\mu+1+\nu}\left(\begin{array}{c}
k \\
l
\end{array}\right)\left(\begin{array}{c}
k \\
l+\nu
\end{array}\right) .
\end{gathered}
$$

Тогда

$$
N_{1 k}-N_{2 k}=\frac{1}{(k !)^{2}} M_{k}
$$

Здесь

$$
M_{k}=\sum_{l=0}^{k}\left[\frac{1}{2 l+\mu+1-\nu}\left(\begin{array}{c}
k \\
l-\nu
\end{array}\right)-\frac{1}{2 l+\mu+1+\nu}\left(\begin{array}{c}
k \\
l+\nu
\end{array}\right)\right]\left(\begin{array}{l}
k \\
l
\end{array}\right) .
$$

Далее выведем формулу для вычисления суммы (19). Предварительно вычислим $M_{0}, M_{1}, M_{2}$ и $M_{3}$, затем определим общий вид этой формулы и методом индукции докажем ее справедливость.

При $k=0$ имеем

$$
M_{0}=\frac{2}{\Gamma(\nu) \Gamma(1-\nu)} \cdot \frac{1}{(\mu+1)^{2}-\nu^{2}} .
$$


Если $k=1$, то получим

$$
M_{1}=\frac{2}{\Gamma(\nu) \Gamma(1-\nu)} \cdot \frac{4}{\left[(\mu+1)^{2}-\nu^{2}\right]\left[(\mu+1+2)^{2}-\nu^{2}\right]} .
$$

Когда $k=2$, вычисления дают следующий результат:

$$
M_{2}=\frac{2 \cdot 4}{\Gamma(\nu) \Gamma(1-\nu)} \cdot \frac{16}{\left[(\mu+1)^{2}-\nu^{2}\right]\left[(\mu+1+2)^{2}-\nu^{2}\right]\left[(\mu+1+4)^{2}-\nu^{2}\right]}
$$

Приведенные выше вычисления для $k=0,1,2$ не дают возможности написать общий вид формулы, поэтому дополнительно найдем

$$
\begin{gathered}
M_{3}=\frac{2 \cdot 36}{\Gamma(\nu) \Gamma(1-\nu)} \cdot \frac{64}{\left[(\mu+1)^{2}-\nu^{2}\right]\left[(\mu+1+2)^{2}-\nu^{2}\right]} \times \\
\quad \times \frac{1}{\left[(\mu+1+4)^{2}-\nu^{2}\right]\left[(\mu+1+6)^{2}-\nu^{2}\right]}= \\
=\frac{2 \cdot(3 !)^{2}}{\Gamma(\nu) \Gamma(1-\nu)} \cdot \frac{2^{2 \cdot 3}}{\left[(\mu+1)^{2}-\nu^{2}\right]\left[(\mu+1+2)^{2}-\nu^{2}\right]} \times \\
\quad \times \frac{1}{\left[(\mu+1+2 \cdot 2)^{2}-\nu^{2}\right]\left[(\mu+1+2 \cdot 3)^{2}-\nu^{2}\right]}
\end{gathered}
$$

Из равенства (20) уже нетрудно записать общий вид формулы для нахождения

$$
M_{k}=\frac{2(k !)^{2}}{\Gamma(\nu) \Gamma(1-\nu)} \cdot \frac{2^{2 k}}{\left[(\mu+1)^{2}-\nu^{2}\right]\left[(\mu+3)^{2}-\nu^{2}\right] \cdots\left[(\mu+1+2 k)^{2}-\nu^{2}\right]} .
$$

Предварительно, используя символ Похгаммера

$$
(a)_{n}=a(a+1) \cdots(a+n-1)=\frac{\Gamma(a+n)}{\Gamma(a)},
$$

преобразуем выражение, входящее в правую часть формулы (21):

$$
\begin{aligned}
& (\mu+1 \pm \nu)(\mu+1+2 \pm \nu) \cdots(\mu+1+2 k \pm \nu)= \\
& =\frac{\mu+1 \pm \nu}{2}\left(\frac{\mu+1 \pm \nu}{2}+1\right) \cdots\left(\frac{\mu+1 \pm \nu}{2}+k\right) 2^{k+1}= \\
& =\left(\frac{\mu+1 \pm \nu}{2}\right)_{k+1} 2^{k+1}=\frac{\Gamma\left(\frac{\mu+1 \pm \nu}{2}+k+1\right) 2^{k+1}}{\Gamma\left(\frac{\mu+1 \pm \nu}{2}\right)} .
\end{aligned}
$$

Тогда формула (21) принимает вид

$$
M_{k}=\frac{(k !)^{2}}{2 \Gamma(\nu) \Gamma(1-\nu)} \cdot \frac{\Gamma\left(\frac{\mu+1+\nu}{2}\right) \Gamma\left(\frac{\mu+1-\nu}{2}\right)}{\Gamma\left(\frac{\mu+1+\nu}{2}+k+1\right) \Gamma\left(\frac{\mu+1-\nu}{2}+k+1\right)} .
$$


Пусть формула (22) верна при произвольном $k$. Докажем ее справедливость при $k+1$. Для этого на основании (19) найдем $M_{k+1}$ и, используя равенство

$$
\left(\begin{array}{l}
a+1 \\
b+1
\end{array}\right)=\left(\begin{array}{l}
a \\
b
\end{array}\right)+\left(\begin{array}{c}
a \\
b+1
\end{array}\right)
$$

разобьем эту величину на четыре суммы:

$$
\begin{aligned}
& M_{k+1}= \\
& =\sum_{l=0}^{k+1}\left[\frac{1}{2 l+\mu+1-\nu}\left(\begin{array}{c}
k \\
l-\nu-1
\end{array}\right)-\frac{1}{2 l+\mu+1+\nu}\left(\begin{array}{c}
k \\
l+\nu-1
\end{array}\right)\right]\left(\begin{array}{c}
k \\
l-1
\end{array}\right)+ \\
& +\sum_{l=0}^{k+1}\left[\frac{1}{2 l+\mu+1-\nu}\left(\begin{array}{c}
k \\
l-\nu
\end{array}\right)-\frac{1}{2 l+\mu+1+\nu}\left(\begin{array}{c}
k \\
l+\nu
\end{array}\right)\right]\left(\begin{array}{c}
k \\
l-1
\end{array}\right)+ \\
& +\sum_{l=0}^{k+1}\left[\frac{1}{2 l+\mu+1-\nu}\left(\begin{array}{c}
k \\
l-\nu-1
\end{array}\right)-\frac{1}{2 l+\mu+1+\nu}\left(\begin{array}{c}
k \\
l+\nu-1
\end{array}\right)\right]\left(\begin{array}{l}
k \\
l
\end{array}\right)+ \\
& +\sum_{l=0}^{k+1}\left[\frac{1}{2 l+\mu+1-\nu}\left(\begin{array}{c}
k \\
l-\nu
\end{array}\right)-\frac{1}{2 l+\mu+1+\nu}\left(\begin{array}{c}
k \\
l+\nu
\end{array}\right)\right]\left(\begin{array}{l}
k \\
l
\end{array}\right)= \\
& =P_{1}+P_{2}+P_{3}+P_{4} \text {. }
\end{aligned}
$$

В силу известных равенств

$$
\begin{gathered}
\left(\begin{array}{c}
k \\
-1
\end{array}\right)=0 \text { и }\left(\begin{array}{c}
k \\
k+1
\end{array}\right)=0 \\
P_{1}=\sum_{l=0}^{k}\left[\frac{1}{2 l+\mu+2+1-\nu}\left(\begin{array}{c}
k \\
l-\nu
\end{array}\right)-\frac{1}{2 l+\mu+2+1+\nu}\left(\begin{array}{c}
k \\
l+\nu
\end{array}\right)\right]\left(\begin{array}{l}
k \\
l
\end{array}\right)= \\
=M_{k}(\text { здесь } \mu \text { надо заменить на } \mu+2)=\left.M_{k}\right|_{\mu=\mu+2}, \quad \\
P_{4}=\sum_{l=0}^{k}\left[\frac{1}{2 l+\mu+1-\nu}\left(\begin{array}{c}
k \\
l-\nu
\end{array}\right)-\frac{1}{2 l+\mu+1+\nu}\left(\begin{array}{c}
k \\
l+\nu
\end{array}\right)\right]\left(\begin{array}{c}
k \\
l
\end{array}\right) \equiv M_{k}, \quad(25) \\
P_{2}=\sum_{l=1}^{k+1}\left[\frac{1}{2 l+\mu+1-\nu}\left(\begin{array}{c}
k \\
l-\nu
\end{array}\right)-\frac{1}{2 l+\mu+1+\nu}\left(\begin{array}{c}
k \\
l+\nu
\end{array}\right)\right]\left(\begin{array}{c}
k \\
l-1
\end{array}\right)= \\
=\sum_{l=0}^{k}\left[\frac{1}{2 l+\mu+3-\nu}\left(\begin{array}{c}
k \\
l+1-\nu
\end{array}\right)-\frac{1}{2 l+\mu+3+\nu}\left(\begin{array}{c}
k \\
l+1+\nu
\end{array}\right)\right]\left(\begin{array}{c}
k \\
l
\end{array}\right), \quad \\
P_{3}=\sum_{l=0}^{k}\left[\frac{1}{2 l+\mu+1-\nu}\left(\begin{array}{c}
k \\
l-\nu-1
\end{array}\right)-\frac{1}{2 l+\mu+1+\nu}\left(\begin{array}{c}
k \\
l+\nu-1
\end{array}\right)\right]\left(\begin{array}{l}
k \\
l
\end{array}\right) . \quad
\end{gathered}
$$


Предварительно на основании (26) и (27) найдем сумму

$$
\begin{aligned}
& P_{2}+P_{3}=\sum_{l=0}^{k}\left[\frac{1}{2 l+\mu+2+1-\nu}\left(\begin{array}{c}
k \\
l+1-\nu
\end{array}\right)-\right. \\
& \left.-\frac{1}{2 l+\mu+2+\nu-1}\left(\begin{array}{c}
k \\
l+\nu-1
\end{array}\right)\right]\left(\begin{array}{l}
k \\
l
\end{array}\right)+ \\
& +\sum_{l=0}^{k}\left[\frac{1}{2 l+\mu+2-1-\nu}\left(\begin{array}{c}
k \\
l-1-\nu
\end{array}\right)-\frac{1}{2 l+\mu+2+1+\nu}\left(\begin{array}{c}
k \\
l+1+\nu
\end{array}\right)\right]\left(\begin{array}{l}
k \\
l
\end{array}\right)= \\
& =-\left.M_{k}\right|_{\substack{\nu=1-\nu \\
\mu=\mu+1}}+\left.M_{k}\right|_{\substack{\nu=1+\nu \\
\mu=\mu+1}} \text {. }
\end{aligned}
$$

Тогда, подставляя (24), (25) и (28) в (23), с учетом формулы (22) получим

$$
\begin{aligned}
& M_{k+1}=\left.M_{k}\right|_{\mu=\mu+2}+M_{k}-\left.M_{k}\right|_{\substack{\nu=1-\nu \\
\mu=\mu+1}}+\left.M_{k}\right|_{\substack{\nu=1+\nu \\
\mu=\mu+1}}= \\
& =\frac{(k !)^{2}}{2 \Gamma(\nu) \Gamma(1-\nu)}\left[\frac{\Gamma\left(\frac{\mu+3+\nu}{2}\right) \Gamma\left(\frac{\mu+3-\nu}{2}\right)}{\Gamma\left(\frac{\mu+3+\nu}{2}+k+1\right) \Gamma\left(\frac{\mu+3-\nu}{2}+k+1\right)}+\right. \\
& +\frac{\Gamma\left(\frac{\mu+1+\nu}{2}\right) \Gamma\left(\frac{\mu+1-\nu}{2}\right)}{\Gamma\left(\frac{\mu+1+\nu}{2}+k+1\right) \Gamma\left(\frac{\mu+1-\nu}{2}+k+1\right)}- \\
& -\frac{\Gamma\left(\frac{\mu+3-\nu}{2}\right) \Gamma\left(\frac{\mu+1+\nu}{2}\right)}{\Gamma\left(\frac{\mu+3-\nu}{2}+k+1\right) \Gamma\left(\frac{\mu+1+\nu}{2}+k+1\right)}- \\
& \left.-\frac{\Gamma\left(\frac{\mu+3+\nu}{2}\right) \Gamma\left(\frac{\mu+1-\nu}{2}\right)}{\Gamma\left(\frac{\mu+3+\nu}{2}+k+1\right) \Gamma\left(\frac{\mu+1-\nu}{2}+k+1\right)}\right]= \\
& =\frac{(k !)^{2}}{2 \Gamma(\nu) \Gamma(1-\nu)}\left[S_{1 k}-S_{2 k}\right] \text {. }
\end{aligned}
$$

Используя формулу $\Gamma(a+1)=a \Gamma(a)$, найдем суммы $S_{1 k}$ и $S_{2 k}$ :

$$
\begin{aligned}
S_{1 k}= & \frac{\Gamma\left(\frac{\mu+1+\nu}{2}\right) \Gamma\left(\frac{\mu+1-\nu}{2}\right)}{\Gamma\left(\frac{\mu+3+\nu}{2}+k+1\right) \Gamma\left(\frac{\mu+3-\nu}{2}+k+1\right)} \times \\
& \quad \times\left[(\mu+1)^{2}-\nu^{2}+(\mu+1+2 k+2)^{2}-\nu^{2}\right]=
\end{aligned}
$$




$$
\begin{gathered}
=\frac{\Gamma\left(\frac{\mu+1+\nu}{2}\right) \Gamma\left(\frac{\mu+1-\nu}{2}\right)\left[(\mu+1)^{2}-\nu^{2}+2(k+1)(\mu+k+2)\right]}{2 \Gamma\left(\frac{\mu+3+\nu}{2}+k+1\right) \Gamma\left(\frac{\mu+3-\nu}{2}+k+1\right)}, \\
S_{2 k}=\frac{\Gamma\left(\frac{\mu+1+\nu}{2}\right) \Gamma\left(\frac{\mu+1-\nu}{2}\right)\left[(\mu+1)^{2}-\nu^{2}+2(k+1)(\mu+1)\right]}{2 \Gamma\left(\frac{\mu+3+\nu}{2}+k+1\right) \Gamma\left(\frac{\mu+3-\nu}{2}+k+1\right)} .
\end{gathered}
$$

Теперь, подставляя (30) и (31) в (29), находим

$$
M_{k+1}=\frac{((k+1) !)^{2}}{2 \Gamma(\nu) \Gamma(1-\nu)} \cdot \frac{\Gamma\left(\frac{\mu+1+\nu}{2}\right) \Gamma\left(\frac{\mu+1-\nu}{2}\right)}{\Gamma\left(\frac{\mu+1+\nu}{2}+k+2\right) \Gamma\left(\frac{\mu+1-\nu}{2}+k+2\right)} .
$$

Таким образом, нами доказано следующее утверждение.

Лемма. Справедлива следующая формула вычисления конечной суммы:

$$
\begin{gathered}
\sum_{l=0}^{k}\left[\frac{1}{2 l+\mu+1-\nu}\left(\begin{array}{c}
k \\
l-\nu
\end{array}\right)-\frac{1}{2 l+\mu+1+\nu}\left(\begin{array}{c}
k \\
l+\nu
\end{array}\right)\right]\left(\begin{array}{l}
k \\
l
\end{array}\right)= \\
=\frac{(k !)^{2}}{2 \Gamma(\nu) \Gamma(1-\nu)} \cdot \frac{\Gamma\left(\frac{\mu+1+\nu}{2}\right) \Gamma\left(\frac{\mu+1-\nu}{2}\right)}{\Gamma\left(\frac{\mu+1+\nu}{2}+k+1\right) \Gamma\left(\frac{\mu+1-\nu}{2}+k+1\right)}
\end{gathered}
$$

Тогда с учетом установленной формулы (32) и соотношений (18), имеем

$$
\begin{gathered}
\widetilde{N}_{\nu}^{(1)}(x)=\frac{x^{\mu+1} \Gamma\left(\frac{\mu+1+\nu}{2}\right) \Gamma\left(\frac{\mu+1-\nu}{2}\right)}{2 \Gamma(\nu) \Gamma(1-\nu)} \times \\
\quad \times \sum_{k=0}^{\infty} \frac{\left(\frac{x^{2}}{4}\right)^{k}}{\Gamma\left(\frac{\mu+1+\nu}{2}+k+1\right) \Gamma\left(\frac{\mu+1-\nu}{2}+k+1\right)}= \\
=\frac{2 \sin \nu \pi \cdot x^{1+\mu}}{\pi\left[(\mu+1)^{2}-\nu^{2}\right]} \sum_{k=0}^{\infty} \frac{\left(\frac{x^{2}}{4}\right)^{k}}{\left(\frac{\mu+3+\nu}{2}\right)_{k}\left(\frac{\mu+3-\nu}{2}\right)}{ }_{k} \\
=\frac{2 \sin \nu \pi \cdot x^{1+\mu}}{\pi\left[(\mu+1)^{2}-\nu^{2}\right]}{ }_{1} F_{2}\left(1 ; \frac{\mu+3+\nu}{2}, \frac{\mu+3-\nu}{2} ; \frac{x^{2}}{4}\right),
\end{gathered}
$$

где ${ }_{1} F_{2}(\cdot)$ - обобщенная гипергеометрическая функция [13, §10.10(IV)].

Тем самым первая задача о вычислении разности (6) выполнена. 
Теперь рассмотрим разность (10). Аналогично вычислению разности (6) с использованием разложения функций $J_{ \pm \nu}(z)$ в обобщенный степенной ряд получим

$$
\widetilde{S}_{\nu}^{(1)}(x)=x^{\mu+1} \sum_{k=0}^{\infty}(-1)^{k}\left(\frac{x}{2}\right)^{2 k}\left(N_{1 k}-N_{2 k}\right)=x^{\mu+1} \sum_{k=0}^{\infty} \frac{(-1)^{k}}{(k !)^{2}}\left(\frac{x}{2}\right)^{2 k} M_{k} .
$$

Подставляя (32) в (34), получим

$$
\begin{gathered}
\widetilde{S}_{\nu}^{(1)}(x)=\frac{2 \sin \nu \pi \cdot x^{1+\mu}}{\pi\left[(\mu+1)^{2}-\nu^{2}\right]} \sum_{k=0}^{\infty} \frac{(-1)^{k}\left(\frac{x^{2}}{4}\right)^{k}}{\left(\frac{\mu+3+\nu}{2}\right)_{k}\left(\frac{\mu+3-\nu}{2}\right)_{k}}= \\
=\frac{x^{1+\mu} 2 \sin \nu \pi}{\pi\left[(\mu+1)^{2}-\nu^{2}\right]}{ }_{1} F_{2}\left(1 ; \frac{\mu+3+\nu}{2}, \frac{\mu+3-\nu}{2} ;-\frac{x^{2}}{4}\right) .
\end{gathered}
$$

Таким образом, полученный нами ряд (35) совпадает с рядом, найденным Ломмелем, как частное решение неоднородного уравнения Бесселя

$$
x^{2} u^{\prime \prime}(x)+x u^{\prime}(x)+\left(x^{2}-\nu^{2}\right) u(x)=x^{\mu+1}
$$

с помощью обобщенного степенного ряда.

Далее вычислим разности (8) и (12). На основании формул

$$
K_{\nu}(z)=\frac{\pi}{2 \sin \nu \pi}\left[I_{-\nu}(z)-I_{\nu}(z)\right], \quad Y_{\nu}(z)=\frac{1}{\sin \nu \pi}\left[J_{\nu}(z) \cos \nu \pi-J_{-\nu}(z)\right]
$$

имеем

$$
\begin{aligned}
& N_{\nu}^{(2)}(x)=\frac{\pi}{2 \sin \nu \pi}\left[I_{\nu}(x) \int_{0}^{x} t^{\mu} I_{-\nu}(t) d t-I_{-\nu}(x) \int_{0}^{x} t^{\mu} I_{\nu}(t) d t\right]= \\
& =\frac{\pi}{2 \sin \nu \pi} \widetilde{N}_{\nu}^{(1)}(x)=\frac{x^{1+\mu}}{(1+\mu)^{2}-\nu^{2}}{ }_{1} F_{2}\left(1 ; \frac{\mu+3+\nu}{2}, \frac{\mu+3-\nu}{2} ; \frac{x^{2}}{4}\right) \\
& S_{\nu}^{(2)}(x)=-\frac{1}{\sin \nu \pi}\left[J_{\nu}(x) \int_{0}^{x} t^{\mu} J_{-\nu}(t) d t-J_{-\nu}(x) \int_{0}^{x} t^{\mu} J_{\nu}(t) d t\right]= \\
& =-\frac{1}{\sin \nu \pi} S_{\nu}^{(1)}(x)= \\
& =-\frac{2 x^{1+\mu}}{\pi\left[(1+\mu)^{2}-\nu^{2}\right]}{ }_{1} F_{2}\left(1 ; \frac{\mu+3+\nu}{2}, \frac{\mu+3-\nu}{2} ;-\frac{x^{2}}{4}\right) .
\end{aligned}
$$

Следовательно, установлено утверждение.

Теорема 1. Справедливы следующие формуль:

$$
I_{\nu}(x) \int_{0}^{x} t^{\mu} I_{-\nu}(t) d t-I_{-\nu}(x) \int_{0}^{x} t^{\mu} I_{\nu}(t) d t=
$$




$$
\begin{gathered}
=\frac{2 x^{1+\mu} \sin \nu \pi}{\pi\left[(1+\mu)^{2}-\nu^{2}\right]}{ }_{1} F_{2}\left(1 ; \frac{\mu+3+\nu}{2}, \frac{\mu+3-\nu}{2} ; \frac{x^{2}}{4}\right), \\
I_{\nu}(x) \int_{0}^{x} t^{\mu} K_{\nu}(t) d t-K_{\nu}(x) \int_{0}^{x} t^{\mu} I_{\nu}(t) d t= \\
=\frac{x^{1+\mu}}{(1+\mu)^{2}-\nu^{2}}{ }_{1} F_{2}\left(1 ; \frac{\mu+3+\nu}{2}, \frac{\mu+3-\nu}{2} ; \frac{x^{2}}{4}\right), \\
=\frac{2 x^{1+\mu} \sin \nu \pi}{\pi\left[(1+\mu)^{2}-\nu^{2}\right]}{ }_{1} F_{2}\left(1 ; \frac{\mu+3+\nu}{2}, \frac{\mu+3-\nu}{2} ;-\frac{x^{2}}{4}\right), \\
J_{\nu}(x) \int_{0}^{x} t^{\mu} J_{-\nu}(t) d t-J_{-\nu}(x) t^{\mu} J_{\nu}(t) d t= \\
J_{\nu}(x) \int_{0}^{x} t^{\mu} Y_{\nu}(t) d t-Y_{\nu}(x) \int_{0}^{x} t^{\mu} J_{\nu}(t) d t= \\
=-\frac{2 x^{1+\mu}}{\pi\left[(1+\mu)^{2}-\nu^{2}\right]}{ }_{1} F_{2}\left(1 ; \frac{\mu+3+\nu}{2}, \frac{\mu+3-\nu}{2} ;-\frac{x^{2}}{4}\right),
\end{gathered}
$$

где $\mu \pm \nu>-1$.

Теперь на основании (33), (35)-(37) с использованием представлений (5), $(7),(9)$ и (11) найдем первоначальные разности (1)-(4):

$$
\begin{aligned}
& N_{\nu}^{(1)}(y)=\frac{y^{2} \sin \nu \pi}{2 \nu \pi}{ }_{1} F_{2}\left(1 ; 1+2 \nu, 1+\nu ; \frac{p^{2}}{4} y^{1 / \nu}\right), \\
& N_{\nu}^{(2)}(y)=\frac{y^{2}}{4 \nu}{ }_{1} F_{2}\left(1 ; 1+2 \nu, 1+\nu ; \frac{p^{2}}{4} y^{1 / \nu}\right), \\
& S_{\nu}^{(1)}(y)=-\frac{y^{2} \sin \nu \pi}{2 \nu \pi}{ }_{1} F_{2}\left(1 ; 1+2 \nu, 1+\nu ;-\frac{p^{2}}{4}(-y)^{1 / \nu}\right), \\
& S_{\nu}^{(2)}(y)=-\frac{y^{2}}{4 \nu}{ }_{1} F_{2}\left(1 ; 1+2 \nu, 1+\nu ;-\frac{p^{2}}{4}(-y)^{1 / \nu}\right),
\end{aligned}
$$

здесь $\nu=1 /(2 q)$.

2. Асимптотические оценки. Поскольку нам неизвестно асимптотическое разложение для функции ${ }_{1} F_{2}(a ; b, c ; \pm z)$ при $z \rightarrow \infty$, воспользуемся ее интегральным представлением [16, форм. (28.96)]:

$$
{ }_{1} F_{2}(a ; b, c ;-z)=\frac{2 \Gamma(b) \Gamma(c)}{\Gamma(a) \Gamma(c-a)} z^{\frac{1-b}{2}} \int_{0}^{1} J_{b-1}(2 t \sqrt{z}) t^{2 a-b}\left(1-t^{2}\right)^{c-a-1} d t,
$$

а также вытекающей из (42) формулой

$$
{ }_{1} F_{2}(a ; b, c ; z)=\frac{2 \Gamma(b) \Gamma(c)}{\Gamma(a) \Gamma(c-a)} z^{\frac{1-b}{2}} \int_{0}^{1} I_{b-1}(2 t \sqrt{z}) t^{2 a-b}\left(1-t^{2}\right)^{c-a-1} d t .
$$


Здесь $a>0, c-a>0$. На их основе найдем интегральные представления для функций (38)-(41):

$$
\begin{aligned}
& N_{\nu}^{(1)}(y)=\frac{2 \nu \Gamma(2 \nu) \sin \nu \pi}{\pi} y\left(\frac{2}{p}\right)^{2 \nu} \int_{0}^{1} I_{2 \nu}\left(t p y^{\frac{1}{2 \nu}}\right) t^{1-2 \nu}\left(1-t^{2}\right)^{\nu-1} d t, y>0, \\
& N_{\nu}^{(2)}(y)=\nu \Gamma(2 \nu) y\left(\frac{2}{p}\right)^{2 \nu} \int_{0}^{1} I_{2 \nu}\left(t p y^{\frac{1}{2 \nu}}\right) t^{1-2 \nu}\left(1-t^{2}\right)^{\nu-1} d t, \quad y>0 \\
& S_{\nu}^{(1)}(y)=\frac{2 \nu \Gamma(2 \nu) \sin \nu \pi}{\pi} y\left(\frac{2}{p}\right)^{2 \nu} \int_{0}^{1} J_{2 \nu}\left(t p(-y)^{\frac{1}{2 \nu}}\right) t^{1-2 \nu}\left(1-t^{2}\right)^{\nu-1} d t, y<0 \\
& S_{\nu}^{(2)}(y)=\nu \Gamma(2 \nu) y\left(\frac{2}{p}\right)^{2 \nu} \int_{0}^{1} J_{2 \nu}\left(t p(-y)^{\frac{1}{2 \nu}}\right) t^{1-2 \nu}\left(1-t^{2}\right)^{\nu-1} d t, \quad y<0
\end{aligned}
$$

В силу формулы асимптотического разложения интеграла, содержащего функцию Бесселя (см. [17, форм. (10.87)], а также [18]), при $b=1, h(t)=(1+t)^{\beta-1}$ имеем

$$
\begin{aligned}
& \int_{0}^{1} J_{\mu}(z t) t^{\alpha-1}\left(1-t^{2}\right)^{\beta-1} d t= \\
= & \frac{2^{\alpha-1} z^{-\alpha}}{\pi} \sum_{k=0}^{\infty} \frac{(-1)^{k}}{k !}\left(\frac{2}{z}\right)^{k} \sin \frac{\pi}{2}(\alpha-\mu-k) A_{k}(\beta) \Gamma\left(\frac{\alpha+k-\mu}{2}\right) \Gamma\left(\frac{\alpha+k+\mu}{2}\right)+ \\
+ & \frac{\sqrt{2}}{\sqrt{\pi}} z^{-\beta-\frac{1}{2}} \sum_{k=0}^{\infty} D_{k}(\alpha, \beta, \mu) \cos \left[z-\frac{\pi}{2}\left(\mu+\beta-k+\frac{1}{2}\right)\right] z^{-k}, \quad z \rightarrow+\infty, \quad(47)
\end{aligned}
$$

где

$$
\begin{gathered}
D_{k}(\alpha, \beta, \mu)=\sum_{j=0}^{k} \frac{\Gamma(\beta+k-j)}{(k-j) !} \frac{\Gamma(1 / 2+\mu+j)}{j ! \Gamma(1 / 2+\mu-j)} d_{j, k-j}(\alpha) 2^{-j}, \\
d_{i k}(\alpha)=\left.\frac{d^{k}}{d b^{k}}\left[b^{a-j-3 / 2}(1+b)^{\beta-1}\right]\right|_{b=1}, \\
A_{k}(\beta)=\left.\frac{d^{k}}{d t^{k}}\left[(b-t)^{\beta-1}(1+b)^{\beta-1}\right]\right|_{\substack{b=1 \\
t=0}} \cdot
\end{gathered}
$$

Из формулы (47) при $z \rightarrow+\infty$ найдем оценку интегралов:

$$
\begin{gathered}
\int_{0}^{1} J_{\mu}(z t) t^{\alpha-1}\left(1-t^{2}\right)^{\beta-1} d t=\frac{2^{\alpha+\beta-2}}{\pi} \sin \frac{\pi}{2}(\alpha-\mu) \Gamma\left(\frac{\alpha-\mu}{2}\right) \Gamma\left(\frac{\alpha+\mu}{2}\right) z^{-\alpha}+ \\
+\frac{2^{\beta-\frac{1}{2}}}{\sqrt{\pi}} \Gamma(\beta) \cos \left[z-\frac{\pi}{2}\left(\mu+\beta+\frac{1}{2}\right)\right] z^{-\beta-\frac{1}{2}}+O\left(z^{-\min \left\{\alpha+1, \beta+\frac{3}{2}\right\}}\right), \quad \\
\int_{0}^{1} I_{\mu}(z t) t^{\alpha-1}\left(1-t^{2}\right)^{\beta-1} d t=\frac{2^{\alpha+\beta-2}}{\pi} \sin \frac{\pi}{2}(\alpha-\mu) i^{-\mu-\alpha} \Gamma\left(\frac{\alpha-\mu}{2}\right) \Gamma\left(\frac{\alpha+\mu}{2}\right) z^{-\alpha}+ \\
+\frac{2^{\beta-\frac{3}{2}} \Gamma(\beta) i^{-\mu-\beta-\frac{1}{2}}}{\sqrt{\pi}}\left[e^{-z} e^{-\frac{i \pi}{2}\left(\mu+\beta+\frac{1}{2}\right)}+e^{z} e^{\frac{i \pi}{2}\left(\mu+\beta+\frac{1}{2}\right)}\right] z^{-\beta-\frac{1}{2}}+O\left(e^{z} z^{-\beta-\frac{3}{2}}\right)=
\end{gathered}
$$




$$
\begin{array}{r}
=\frac{2^{\beta-\frac{3}{2}} \Gamma(\beta)}{\sqrt{\pi}} \exp \left[\frac{i \pi}{2}\left(\mu+\beta+\frac{1}{2}\right)-\left(\mu+\beta+\frac{1}{2}\right) \ln i\right] e^{z} z^{-\beta-\frac{1}{2}}+ \\
+O\left(e^{z} z^{-\beta-\frac{3}{2}}\right) .
\end{array}
$$

Тогда в силу оценок (49) и (48) из (43)-(46) при $p \rightarrow+\infty$ получим

$$
\begin{aligned}
N_{\nu}^{(1)}(y)=\frac{2^{3 \nu-\frac{1}{2}} \nu \Gamma(\nu) \Gamma(2 \nu)}{\pi \sqrt{\pi} \nu \pi} \exp \left[\left(3 \nu+\frac{1}{2}\right)\left(\frac{i \pi}{2}-\ln i\right)\right] \times \\
\times y^{\frac{1}{2}-\frac{1}{4 \nu}} p^{-3 \nu-\frac{1}{2}} e^{p y^{1 /(2 \nu)}}+O\left(e^{p y^{1 /(2 \nu)}} y^{-3 \nu-\frac{3}{2}}\right), \\
N_{\nu}^{(2)}(y)=\frac{2^{3 \nu-\frac{3}{2}} \nu \Gamma(\nu) \Gamma(2 \nu)}{\sqrt{\pi}} \exp \left[\left(3 \nu+\frac{1}{2}\right)\left(\frac{i \pi}{2}-\ln i\right)\right] \times \\
\times y^{\frac{1}{2}-\frac{1}{4 \nu}} p^{-3 \nu-\frac{1}{2}} e^{p y^{1 /(2 \nu)}}+O\left(e^{p y^{1 /(2 \nu)}} y^{-3 \nu-\frac{3}{2}}\right),
\end{aligned}
$$

$$
\begin{aligned}
S_{\nu}^{(1)}(y)=\pi^{-2} 2^{2+\nu} \nu^{2} \Gamma(2 \nu) \Gamma(-2 \nu) \sin \nu \pi \sin 2 \nu \pi(-y)^{2-\frac{1}{\nu}} p^{-2}- \\
-\pi^{-\frac{3}{2}} 2^{3 \nu+\frac{1}{2}} \nu \Gamma(\nu) \Gamma(2 \nu) \sin \nu \pi \cos \left[p(-y)^{\frac{1}{2 \nu}}-\frac{\pi}{2}\left(3 \nu+\frac{1}{2}\right)\right] \times \\
\times(-y)^{\frac{1}{2}-\frac{1}{4 \nu}} p^{-3 \nu-\frac{1}{2}}+O\left(p^{-\min \left\{3,3 \nu+\frac{3}{2}\right\}}\right)= \\
=-\pi^{- \text {frac } 32} 2^{3 \nu+\frac{1}{2}} \nu \Gamma(\nu) \Gamma(2 \nu) \sin \nu \pi \cos \left[p(-y)^{\frac{1}{2 \nu}}-\frac{\pi}{2}\left(3 \nu+\frac{1}{2}\right)\right] \times \\
\times(-y)^{\frac{1}{2}-\frac{1}{4 \nu}} p^{-3 \nu-\frac{1}{2}}+O\left(p^{-2}\right),
\end{aligned}
$$

$$
\begin{aligned}
& S_{\nu}^{(2)}(y)=\pi^{-1} 2^{1+\nu} \nu^{2} \Gamma(2 \nu) \Gamma(-2 \nu) \sin 2 \nu \pi(-y)^{2-\frac{1}{\nu}} p^{-2}- \\
&-\pi^{-\frac{1}{2}} 2^{3 \nu-\frac{1}{2}} \nu \Gamma(\nu) \Gamma(2 \nu) \cos [\left.p(-y)^{\frac{1}{2 \nu}}-\frac{\pi}{2}\left(3 \nu+\frac{1}{2}\right)\right] \times \\
& \times(-y)^{\frac{1}{2}-\frac{1}{4 \nu}} p^{-3 \nu-\frac{1}{2}}+O\left(p^{-\min \left\{3,3 \nu+\frac{3}{2}\right\}}\right)= \\
&=-\pi^{-\frac{1}{2}} 2^{3 \nu-\frac{1}{2}} \nu \Gamma(\nu) \Gamma(2 \nu) \cos \left[p(-y)^{\frac{1}{2 \nu}}-\frac{\pi}{2}\left(3 \nu+\frac{1}{2}\right)\right] \times \\
& \times(-y)^{\frac{1}{2}-\frac{1}{4 \nu}} p^{-3 \nu-\frac{1}{2}}+O\left(p^{-2}\right) .
\end{aligned}
$$

Таким образом, нами доказана следующая основная

Теорема 2. Для разностей (1)-(4) при $p \rightarrow+\infty$ справедливы соответственно асимптотические оченки (50)-(53).

Конкурирующие интересы. Конкурирующих интересов не имею.

Авторский вклад и ответственность. Я несу полную ответственность за предоставление окончательной версии рукописи в печать. Окончательная версия рукописи мною одобрена.

Финансирование. Работа выполнена при финансовой поддержке РФФИ (проект № 17-41-020516). 


\section{Библиографический список}

1. Сабитов К. Б., Рахманова Л. Х. Начально-граничная задача для вырождающегося уравнения смешанного параболо-гиперболического типа в прямоугольной области // Диффер. уравн., 2008. Т. 44, № 9. С. 1175-1181.

2. Сабитова Ю. К. Нелокальные начально-граничные задачи для вырождающегося гиперболического уравнения // Изв. вузов. Матем., 2009. № 12. С. 49-58.

3. Бурханова (Хаджи) И. А. Критерий единственности решения обратной задачи уравнения смешанного типа с оператором типа Чаплыгина / Дифферени. уравнения и смежные проблемы: Тр. междун. научн. конф.; в 2-х т. Т. 1. Уфа: БашГУ, 2013. С. 140-144.

4. Сабитов К. Б., Сидоров С. Н. Об одной нелокальной задаче для вырождающегося параболо-гиперболического уравнения // Диффер. уравн., 2014. Т. 50, № 3. С. 356-365.

5. Сабитова Ю. К. Краевая задача с нелокальным интегральным условием для уравнений смешанного типа с вырождением на переходной линии // Матем. заметки, 2015. Т. 98, № 3. C. 393-406. doi : 10.4213/mzm9135.

6. Сабитов К. Б., Сидоров С. Н. Обратная задача для вырождающегося парабологиперболического уравнения с нелокальным граничным условием // Изв. вузов. Матем., 2015. № 1. С. 46-59.

7. Мартемьянова Н. В. Необходимое и достаточное условие единственности решения нелокальной обратной задачи для уравнения типа Чаплыгина / Mатематическое моделирование прочессов и систем: Материалы V Всерос. науч.-практ. конф., приуроченной к 110-летию со дня рождения академика А. Н. Тихонова (17-19 ноября 2016 г., г. Стерлитамак), Ч. ІІІ. Стерлитамак: Стерлитамакский филиал БашГУ, 2016. С. 19-23.

8. Сабитова Ю. К. Задача Дирихле для уравнения гиперболического типа со степенным вырождением в прямоугольной области // Диффер. уравн., 2018. Т. 54, № 2. С. 228-238. doi : 10.1134/S0374064118020097.

9. Сабитов К. Б., Сидоров С. Н. Начально-граничная задача для неоднородных вырождающихся уравнений смешанного параболо-гиперболического типа / Дифференциалънъе уравнения. Математическая физика/ Итоги науки и техн. Сер. Соврем. мат. и ее прил. Темат. обз., Т. 137. Москва: ВИНИТИ РАН, 2017. С. 26-60.

10. Сидоров С. Н. Обратные задачи для уравнения смешанного парабологиперболического типа с вырождающейся параболической частью// Сиб. электрон. матем. изв., 2019. Т. 16. С. 144-157. doi: 10.33048/semi.2019.16.007.

11. Von Lommel E. Ueber eine mit den Bessel'schen Functionen verwandte Function // Math. Ann., 1875. vol. 9, no. 3. pp. 425-444. doi : 10.1007/bf01443342.

12. Watson G. N. A treatise on the theory of Bessel functions. Cambridge: Cambridge Univ. Press, 1944. vi+804 pp.

13. Erdélyi A., Magnus W., Oberhettinger F., Tricomi F. G. Higher transcendental functions. vol. II / Bateman Manuscript Project. New York, Toronto, London: McGraw-Hill Book Co.. xvii +396 pp.

14. Сабитов К. Б. Вычисление определенных интегралов от произведения бесселевых функций // Вестн. МГУ, Сер. 15. Вычисл. матем. и киберн., 1992. № 1. С. 24-29.

15. Сабитов К. Б. Построение в явном виде решений задач Дарбу для телеграфного уравнения и их применение при обращении интегральных уравнений. II // Диффер. уравн., 1992. Т. 28, № 7. С. 1138-1145.

16. Риекстыньш Э. Я. Асимптотические разложения интегралов. Т. 3. Рига: Зинатне, 1981. $370 \mathrm{c}$.

17. Риекстыньш Э. Я. Асимптотические разложения интегралов. Т.1. Рига: Зинатне, 1974. 392 c.

18. Тихонов А. Н. Об асимптотическом поведении интегралов, содержащих бесселевы функции // Докл. АН СССР, 1959. Т. 125, № 5. С. 982-985. 


\title{
MSC: 33C20
}

\section{Asymptotic estimates of the difference of products of Bessel functions by the integral of these functions}

\author{
K. B. Sabitov \\ 1 Sterlitamak branch of the Bashkir State University, \\ Faculty of Mathematics and Information Technology, \\ 37, Lenina prospekt, Sterlitamak, 453103, Russian Federation. \\ 2 Institute of Strategic Studies of Bashkortostan Republic, Sterlitamak Branch, \\ 68, Odesskaya str., Sterlitamak, 453103, Russian Federation.
}

\begin{abstract}
In the study of direct and inverse problems of finding the right-hand side of degenerate equations of mixed type with different boundary conditions, the problem arises of establishing asymptotic estimates for the differences of the products of cylindrical functions by the integral of these functions. Previously, on the basis of the established new formula for finding the finite binomial sum, the differences between the products of cylindrical functions and a definite integral of these functions are calculated through a generalized hypergeometric function. Using the asymptotic formula for large values of the argument for the generalized hypergeometric function, asymptotic estimates are established for large values of the parameter for the indicated differences of the Bessel functions of the first and second kind, as well as for modified Bessel functions.
\end{abstract}

Keywords: Bessel functions, modified Bessel functions, integrals of Bessel functions, finite binomial sum, generalized hypergeometric function, asymptotic estimates.

Received: $3^{\text {rd }}$ April, 2019 / Revised: $16^{\text {th }}$ August, $2019 /$

Accepted: $16^{\text {th }}$ September, $2019 /$ First online: $21^{\text {st }}$ November, 2019

Competing interests. I declare that I have no competing interests.

Author's Responsibilities. I take full responsibility for submitting the final manuscript in print. I approved the final version of the manuscript.

Funding. This work was supported by the Russian Foundation for Basic Research (project no. 17-41-020516).

\section{Research Article}

( () (i) The content is published under the terms of the Creative Commons Attribution 4.0 International License (http://creativecommons.org/licenses/by/4.0/)

Please cite this article in press as:

Sabitov K. B. Asymptotic estimates of the difference of products of Bessel functions by the integral of these functions, Vestn. Samar. Gos. Tekhn. Univ., Ser. Fiz.-Mat. Nauki [J. Samara State Tech. Univ., Ser. Phys. Math. Sci.], 2020, vol. 24, no. 1, pp. 41-55. doi: 10.14498/vsgtu1685 (In Russian).

\section{Author's Details:}

Kamil B. Sabitov (1) https://orcid.org/0000-0001-9516-2704

Dr. Phys. \& Math. Sci., Professor; Head of Mathematical Analysis Dept. ${ }^{1}$; Head of Applied Mathematics and Informatics Lab. ${ }^{2}$; e-mail: sabitov_fmf@mail.ru 


\section{References}

1. Sabitov K. B., Rakhmanova L. Kh. Initial-boundary value problem for an equation of mixed parabolic-hyperbolic type in a rectangular domain, Differ. Equ., 2008, vol.44, no. 9, pp. 1218-1224. doi : 10.1134/S0012266108090036.

2. Sabitova Yu. K. Nonlocal initial-boundary-value problems for a degenerate hyperbolic equation, Russian Math. (Iz. VUZ), 2009, vol.53, no.12, pp. 41-49. doi:10.3103/ S1066369X09120068.

3. Burkhanova (Khadzhi) I. A. An uniqueness criterion for solving of the inverse problem of mixed-type equation with Chaplygin type operator, In: Differents. uravneniia i smezhnye problemy [Differential Equations and Related Problems], vol.1. Ufa, Bashkir State Univ., 2013, pp. 140-144 (In Russian).

4. Sabitov K. B., Sidorov S. N. On a nonlocal problem for a degenerating parabolic-hyperbolic equation, Differ. Equ., 2014, vol. 50, no. 3, pp. 352-361. doi: 10.1134/S0012266114030094.

5. Sabitova Yu. K. Boundary-value problem with nonlocal integral condition for mixed-type equations with degeneracy on the transition line, Math. Notes, 2015, vol. 98, no. 3, pp. 454465. doi: $10.1134 /$ S0001434615090114.

6. Sabitov K. B., Sidorov S. N. Inverse problem for degenerate parabolic-hyperbolic equation with nonlocal boundary condition, Russian Math. (Iz. VUZ), 2015, vol. 59, no. 1, pp. 39-50. doi : 10.3103/S1066369X15010041.

7. Martem'yanova N. V. A necessary and sufficient condition for the uniqueness of a solution of nonlocal inverse problem for Chaplygin-type equation, In: Matematicheskoe modelirovanie protsessov $i$ sistem [Mathematical modeling of processes and systems] (November 17-19, 2016, Sterlitamak), Part. III. Sterlitamak, Sterlitamak branch of Bashkir State Univ., 2016, pp. 19-23 (In Russian).

8. Sabitova Y. K. The Dirichlet problem for a hyperbolic-type equation with power degeneracy in a rectangular domain, Differ. Equ., 2018, vol.54, no. 2, pp. 228-238. doi: 10.1134/ S001226611802009X.

9. Sabitov K. B., Sidorov S. N. Initial-boundary-value problem for inhomogeneous degenerate equations of mixed parabolic-hyperbolic type, J. Math. Sci. (N. Y.), 2019, vol. 236, no.6, pp. 603-640. doi : 10.1007/s10958-018-4136-y.

10. Sidorov S. N. Inverse problems for a mixed parabolic-hyperbolic equation with a degenerate parabolic part, Sib. Elektron. Math. Reports, 2019, vol.16, pp. 144-157 (In Russian). doi : 10.33048/semi.2019.16.007.

11. Von Lommel E. Ueber eine mit den Bessel'schen Functionen verwandte Function, Math. Ann., 1875, vol. 9, no. 3, pp. 425-444. doi : 10.1007/bf01443342.

12. Watson G. N. A treatise on the theory of Bessel functions. Cambridge, Cambridge Univ. Press, 1944, vi+804 pp.

13. Erdélyi A., Magnus W., Oberhettinger F., Tricomi F. G. Higher transcendental functions, vol. II, Bateman Manuscript Project. New York, Toronto, London, McGraw-Hill Book Co., xvii+396 pp.

14. Sabitov K. B. Calculating definite integrals of products of Bessel functions, Mosc. Univ. Comput. Math. Cybern., 1992, vol. 47, pp. 26-32.

15. Sabitov K. B. Construction in explicit form of solutions of the Darboux problems for the telegraph equation and their application in the inversion of integral equations. II, Differ. Equ., 1992, vol. 28, no. 7, pp. 901-908.

16. Riekstiṇš E. J. Asimptoticheskie razlozheniia integralov [Asymptotic Expansions of Integrals], vol. 3. Riga, Zinatne, 1981, 370 pp. (In Russian)

17. Riekstiṇš E. J. Asimptoticheskie razlozheniia integralov [Asymptotic Expansions of Integrals], vol. 1. Riga, Zinatne, 1974, 392 pp. (In Russian)

18. Tikhonov A. N. The asymptotic behaviour of integrals containing Bessel functions, Dokl. Akad. Nauk SSSR, 1959, vol. 125, no. 5, pp. 982-985 (In Russian). 